\title{
Optimización energética en la fabricación de baldosas cerámicas mediante el uso de aceite térmico
}

\author{
A. MEZQUITA(1), E. MONFORT(1), E. VAQUER ${ }^{(1)}$, S. FERRER ${ }^{(1)}$, M.A. ARNAL ${ }^{(2)}$, J. TOLEDO ${ }^{(3)}$, M.A. CUESTA ${ }^{(3)}$ \\ (1) Instituto de Tecnología Cerámica (ITC). Asociación de Investigación de las Industrias Cerámicas (AICE). Universitat Jaume I. Castellón. España. \\ ${ }^{(2)}$ Azulev, S.A.U. Onda. España. \\ (3) Endesa Energía, S.A.U. Madrid. España.
}

\begin{abstract}
El proceso de fabricación de baldosas cerámicas consume gran cantidad de energía, mayoritariamente energía térmica, que se obtiene de la combustión de gas natural. El incremento del coste de este combustible, así como la situación económica actual, hace que este coste sea crítico para las empresas y pueda mermar su competitividad.

El proceso de cocción de las baldosas cerámicas en hornos de rodillos no destaca precisamente por el aprovechamiento de la energía utilizada, pues aproximadamente el $50 \%$ se pierde a través de las chimeneas de humos y de enfriamiento del horno. Con el objetivo de mejorar el aprovechamiento de la energía consumida durante la operación de cocción se han instalado, en las chimeneas de un horno, dos intercambiadores de calor, en los cuales los gases procedentes del horno ceden su calor sensible a un aceite térmico, que posteriormente lo transfiere a través de otros dos intercambiadores a los gases de secado en las conducciones de recirculación de un secadero vertical.

En este trabajo se presenta una planta industrial experimental en la que los resultados obtenidos de ahorro energético se sitúan en un valor medio de $650 \mathrm{~kW}$, dependiendo de las condiciones de funcionamiento y materiales procesados.
\end{abstract}

Palabras clave: eficiencia energética, recuperación de calor, intercambio de calor, aceite térmico.

Energy optimization in ceramic tile manufacture by using thermal oil

The ceramic tile manufacturing process consumes a great amount of energy, mainly thermal energy, which is obtained from natural gas combustion. The increased cost of this fuel and the current economic situation make cost a critical issue that can hurt company competitiveness.

The ceramic tile firing process in roller kilns does not exactly stand out for its energy efficiency, because about $50 \%$ of the energy input is lost through the kiln combustion flue gas and cooling gas stacks.

With a view to improving the reuse of the energy consumed in the firing operation, two heat exchangers were installed in the stacks of a kiln. In these heat exchangers, the kiln gases transfer their sensible heat to a thermal oil that then passes this on, through two other exchangers, to the drying gases in the recirculation ducts of a vertical dryer.

This study presents an experimental industrial plant in a fine-tuning test phase, in which the preliminary results indicate an energy efficiency improvement in a range of $60-90 \%$, depending on the operating conditions and processed materials.

Keywords: energy efficiency, heat recovery, heat Exchange, thermal oil

\section{INTRODUCCIÓN}

El proceso de fabricación de baldosas cerámicas requiere un elevado consumo de energía, principalmente de energía térmica.

Todas las fases del proceso de fabricación consumen energía eléctrica. El consumo de energía térmica se produce principalmente en tres etapas: secado de las suspensiones cerámicas por atomización, secado de los soportes, y cocción de las baldosas. La energía térmica empleada en el proceso se obtiene, principalmente, por la combustión de gas natural.

En la figura 1 se muestra esquemáticamente, el proceso de fabricación de baldosas cerámicas.

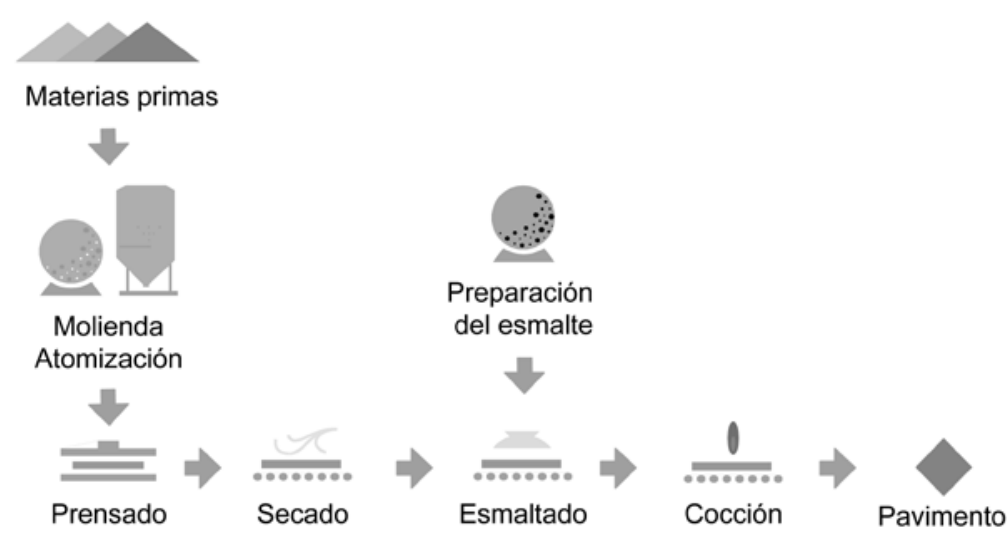

Figura 1. Proceso de fabricación de baldosas cerámicas. 
La cocción de baldosas cerámicas por monococción en España se realiza en hornos de rodillos que utilizan gas natural como fuente de energía térmica. El consumo medio de energía en esta etapa del proceso se cifra en torno a 705 $\mathrm{kWh} / \mathrm{t}$ producto cocido, de los cuales entre un 5 y un $20 \%$ se aprovechan en la cocción del producto, y el resto se pierde por las chimeneas del horno, a través de las paredes y fisuras del horno y con las piezas que salen del horno. En la figura 2 se muestra un diagrama de Sankey típico de un horno de rodillos, en el cual se representa de manera esquemática el aporte porcentual de cada una de las corrientes que intervienen en el balance de energía al horno.

Se aprecia que aproximadamente el $50 \%$ de la energía aportada al proceso de cocción se pierde a través de las chimeneas (de humos de combustión y de gases de enfriamiento).

El objeto de este trabajo es precisamente aprovechar esta energía mediante la instalación de un sistema de recuperación de calor.

En la actualidad en los hornos de cocción de baldosas cerámicas se aprovecha, mayoritariamente, el calor residual de los gases procedentes la chimenea de enfriamiento. Estos gases están compuestos por aire exento de contaminantes, ya que son los gases resultantes del contacto directo del aire utilizado para reducir la temperatura de las piezas en la zona de enfriamiento.

Sin embargo, debido a que durante la cocción de las piezas cerámicas, éstas experimentan reacciones químicas, los gases procedentes de la chimenea de humos poseen contaminantes, pues contienen los productos derivados de la combustión del gas natural y de las reacciones químicas del material producidas durante el proceso de cocción. Para poder aprovechar estos gases es necesario un tratamiento previo a su utilización.

Las posibilidades para aprovechar el calor residual de los gases de combustión son:

\section{- Instalación de un sistema de depuración}

Existe la posibilidad de depurar los gases de combustión y aprovecharlos, junto con los de la zona de enfriamiento, en los secaderos.

Esta opción tiene como factor limitante la temperatura de los gases, ya que los sistemas de depuración utilizados, los filtros de mangas, habitualmente no pueden sobrepasar temperaturas del orden de $180-200{ }^{\circ} \mathrm{C}$, dependiendo del tipo de material de las mangas.

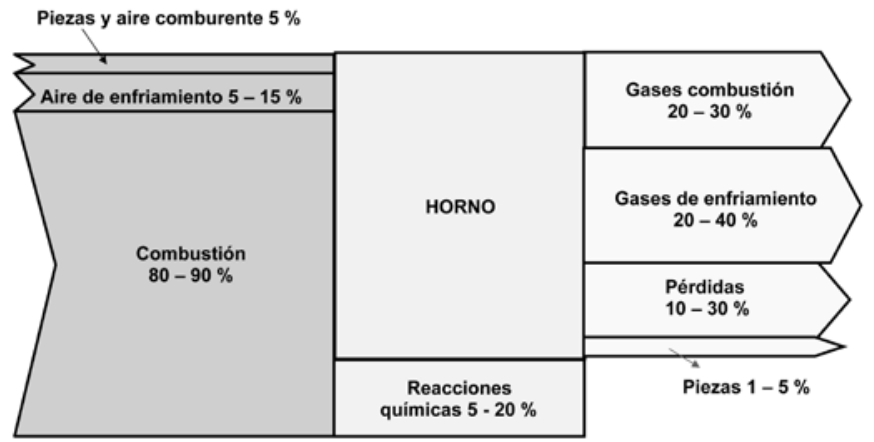

Figura 2. Diagrama de Sankey estándar de un horno de cocción de baldosas.

\section{- Instalación de intercambiadores de calor}

Otra posibilidad consiste en la instalación de intercambiadores de calor con un fluido intermedio que transporte el calor desde el horno hasta el secadero.

Esta es la opción planteada en el siguiente estudio, que además tiene la ventaja de que, puesto que tras el intercambiador de calor la temperatura de los gases de combustión desciende, es posible instalar un sistema de depuración de los mismos, sin necesidad de diluirlos previamente para reducir su temperatura.

\section{OBJETIVO}

El objetivo de este estudio es mejorar el aprovechamiento de la energía consumida en la operación de cocción, mediante la instalación de un novedoso sistema de recuperación de calor, que permite reducir las pérdidas de calor a través de las chimeneas y aprovechar esta energía en la etapa de secado.

\section{DESCRIPCIÓN DEL PROCESO}

\subsection{Descripción de la instalación}

La instalación consiste en una recuperación de calor, basada en un anillo de aceite térmico que, mediante dos intercambiadores de aceite/aire instalados en las chimeneas de humos de combustión y de aire de enfriamiento del horno, capta calor de los gases, reduciendo por tanto, la temperatura final de estos y no su caudal, de forma que la energía se transfiere desde el horno hasta el secadero utilizando un aceite como fluido térmico. El aceite térmico calentado en los dos intercambiadores del horno se une en una única corriente hasta el secadero.

La conducción por la que el aceite térmico circula desde el horno hasta el secadero está calorifugada, para minimizar las pérdidas energéticas. El calor en los secaderos se cede a los gases de secado a través de dos intercambiadores aceite/aire adicionales situados en las dos recirculaciones, no aportando caudal de aire adicional a los secaderos.

El aceite térmico se utiliza en un circuito cerrado, de forma que tras ceder calor a los gases del secadero vuelve hasta los intercambiadores de calor del horno para iniciar de nuevo el proceso. En este circuito existe un sistema de válvulas con bypass, que permiten mantener la temperatura del aceite en el valor óptimo, de forma que aumenta la eficiencia global del proceso.

En función de las necesidades del sistema el aceite térmico cede el calor, preferente a una o a las dos recirculaciones de los gases de secado.

En la figura 3 se muestra un esquema general de la instalación.

\subsection{Descripción de los elementos principales}

\subsubsection{SECADERO}

El secadero estudiado es un secadero vertical; el aporte de calor con anterioridad a la actuación propuesta se realiza mediante la combustión de gas natural a través de dos quemadores situados en las dos recirculaciones de aire. 


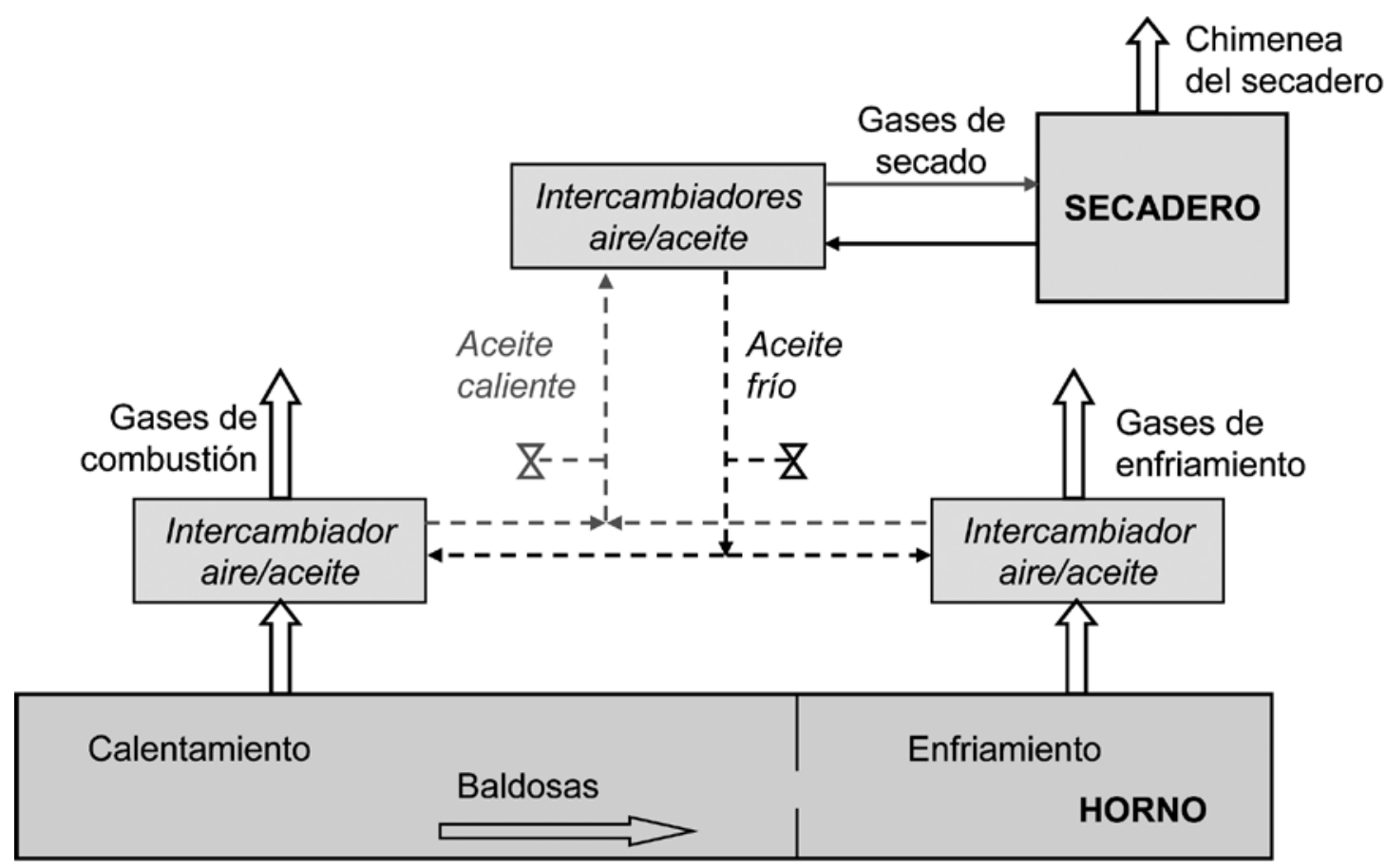

Figura 3. Esquema general de la instalación de recuperación de calor.

En la figura 4 se ha muestra un esquema del secadero estudiado.

La circulación de las piezas, al entrar al secadero, es en primer lugar ascendente, y tras pasar por la zona alta del secadero, descienden hacia la zona de estabilización.

Tras entrar en contacto con las piezas, una parte de los gases de secado se recircula de nuevo hacia el secadero mediante dos recirculaciones independientes, donde están ubicados los quemadores vena de aire, y el resto se vierte a la atmósfera a través de la chimenea. En estas dos recirculaciones es donde se han ubicado los dos intercambiadores de calor de aceite que calientan los gases de secado.
Las temperaturas de consigna de los quemadores se establecen para los gases de secado que salen del secadero, de modo que los elementos calefactores (quemadores e intercambiadores de calor) calientan los gases que se introducen en el secadero tanto como sea necesario para que en la salida, la temperatura sea la establecida.

El aceite llega hasta el secadero a una temperatura de $200{ }^{\circ} \mathrm{C}$, donde se reparte automáticamente a los dos intercambiadores. Tras el intercambio de calor con los gases de secado, el aceite retorna hacia el horno a una temperatura aproximada de $170{ }^{\circ} \mathrm{C}$.

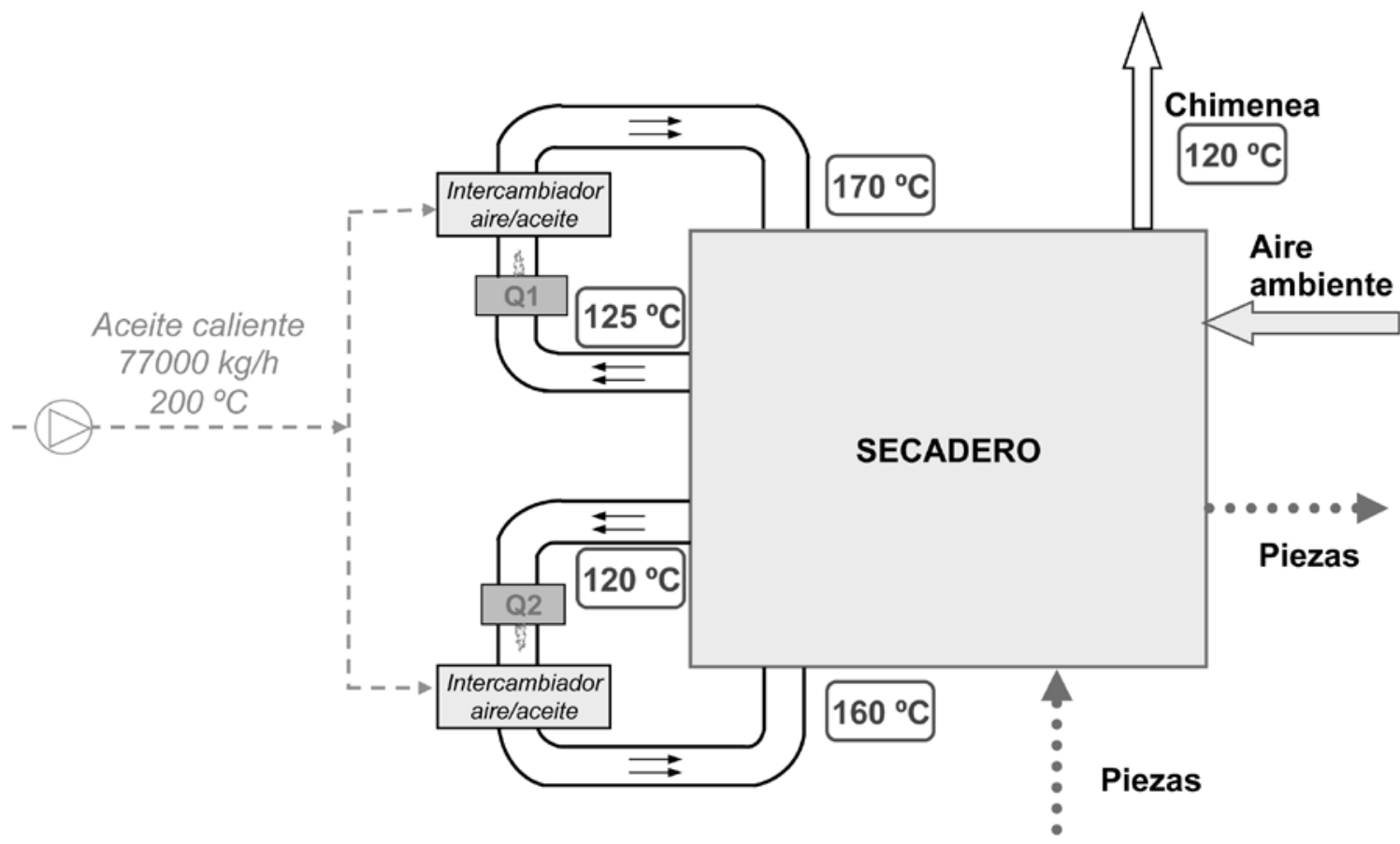

Figura 4. Esquema del secadero. 

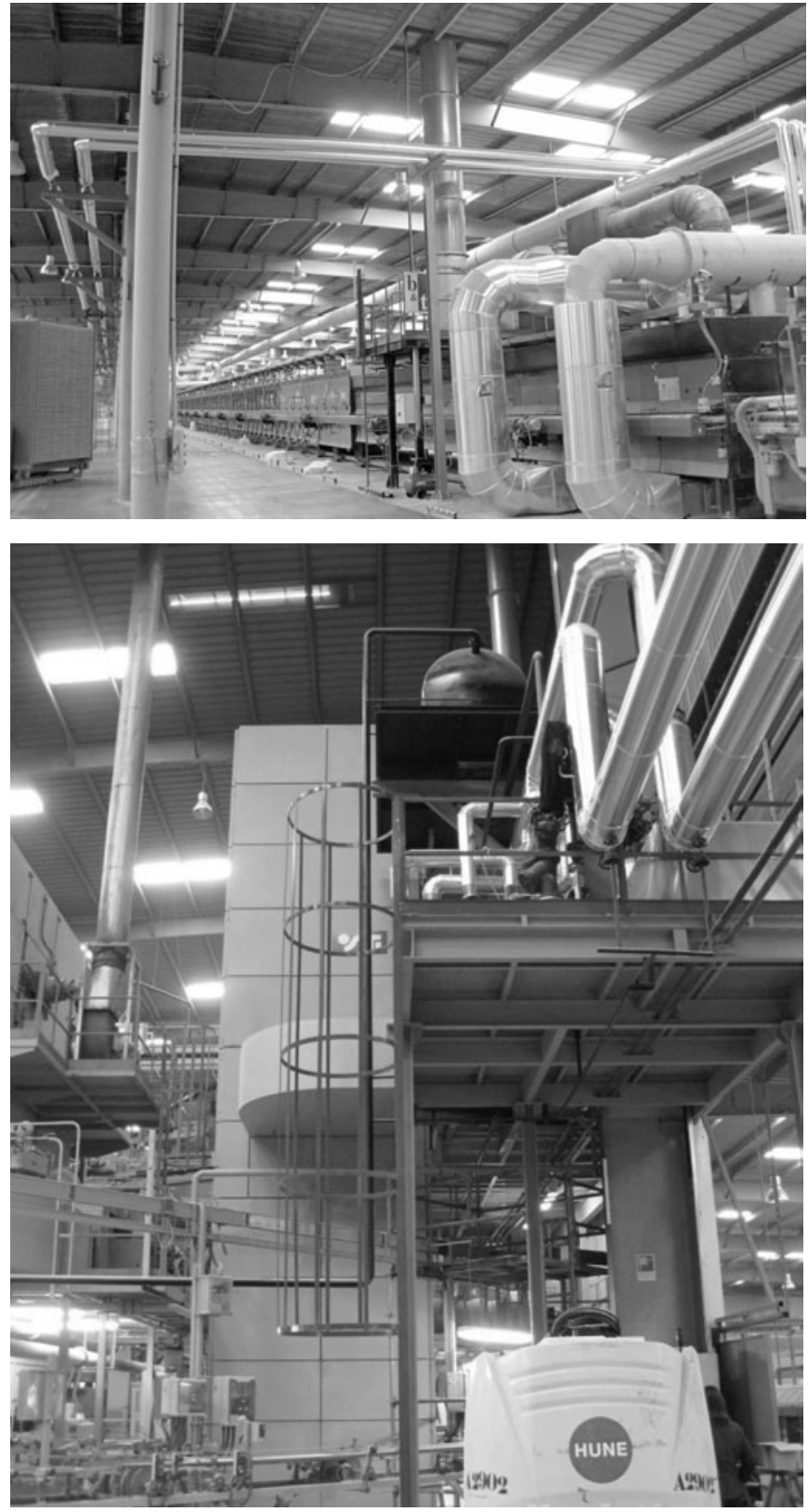

Figura 5. Conducto calorifugado que transporta el aceite térmico hacia el secadero (arriba) y llegada del conducto de aceite térmico al secadero (abajo).

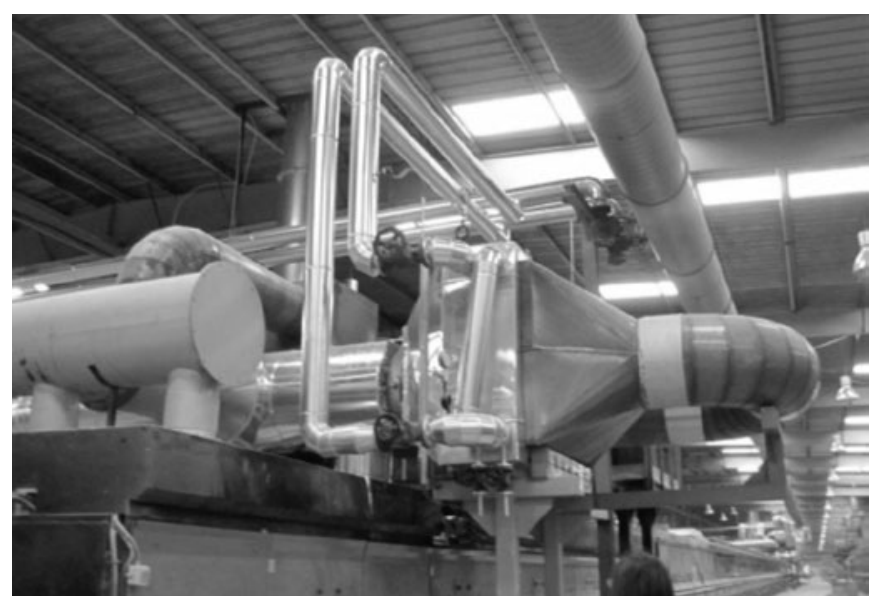

Figura 6. Intercambiador aceite/aire en la chimenea de los gases de combustión.
En la figura 5 se muestran dos fotografías de la conducción por la que circula el aceite desde el horno hasta el secadero.

\subsubsection{HORNO}

El horno estudiado es un horno monoestrato de rodillos, dividido en tres zonas: calentamiento, cocción y enfriamiento.

El aporte de calor se realiza mediante la combustión de gas natural en quemadores de alta velocidad, distribuidos a lo largo de las paredes laterales del horno por encima y por debajo del plano que delimitan los rodillos, en las zonas de calentamiento y cocción.

Previamente a la instalación del sistema de recuperación, los gases de combustión del horno, tras diluirse con aire ambiente, se emitían directamente a la atmósfera. Tras la instalación del sistema de recuperación, éstos no se diluyen con aire ambiente, y transfieren calor al fluido térmico. En la figura 6 se muestra el intercambiador aceite/aire instalado en la salida de los gases de combustión del horno.

Los gases de enfriamiento se recogen de las zonas de enfriamiento rápido, enfriamiento indirecto y enfriamiento final. De esta chimenea, se recupera una parte a un secadero prehorno, otra a los quemadores del horno y el resto se vierte a la atmósfera.

El intercambiador de calor de la zona de enfriamiento se ha instalado en la conducción de recuperación de los gases procedentes del enfriamiento rápido, ya que son los que poseen mayor temperatura.

\subsubsection{INTERCAMBIADORES DE CALOR Y FLUIDO TÉRMICO}

En los cuatro intercambiadores de calor el aceite térmico circula en contracorriente con los gases procedentes del horno, para alcanzar una mayor eficiencia energética.

El aceite refinado se ha formulado especialmente como fluido de intercambio térmico, y se ha aditivado convenientemente para proporcionarle estabilidad térmica y resistencia a la oxidación. De este modo, se comporta de forma adecuada frente a los aumentos de temperatura ocurridos durante su utilización y al espaciamiento de los intervalos de cambio de aceite.

TABla 1. CARACTERí́sticAs DEL ACEITE.

\begin{tabular}{|c|c|c|}
\hline Propiedad & Norma & Valor \\
\hline Temperatura máxima de operación $\left({ }^{\circ} \mathrm{C}\right)$ & --- & 350 \\
\hline Viscosidad cinemática a $40{ }^{\circ} \mathrm{C}(\mathrm{cSt})$ & ASTM D 445 & $20-25$ \\
\hline Índice de viscosidad & ASTM D 2270 & 95 \\
\hline Densidad a $15{ }^{\circ} \mathrm{C}\left(\mathrm{g} / \mathrm{cm}^{3}\right)$ & ASTM D 1298 & 0,860 \\
\hline Punto de inflamación $\left({ }^{\circ} \mathrm{C}\right)$ & ASTM D 92 & 210 \\
\hline Punto de congelación $\left({ }^{\circ} \mathrm{C}\right)$ & ASTM D 97 & -14 \\
\hline
\end{tabular}


Sus bases refinadas, le proporcionan a este termo-fluido una elevada resistencia térmica así como propiedades antioxidantes, lo que hace disminuir considerablemente la formación de insolubles y depósitos sobre las tuberías asegurando así la perfecta circulación y transmisión de calor del fluido, evitando la obstrucción de los circuitos.

Además, este fluido presenta una viscosidad baja que permite el arranque inmediato a bajas temperaturas, óptima transmisión de calor y ofrece así un mayor rendimiento de la bomba. Con todo esto, permite garantizar un perfecto funcionamiento y un alto rendimiento en las instalaciones de tipo cerrado sin contacto directo con el aire y dotadas de medios mecánicos de circulación.

Las propiedades de aceite térmico se muestran en la Tabla 1.

\section{PROCEDIMIENTO EXPERIMENTAL}

\subsection{Determinación del ahorro energético}

La medida y verificación del ahorro se ha realizado según la metodología descrita en el protocolo internacional de medida y verificación (IPMVP) promovido por la Efficiency Valuation Organization (EVO).

La Medida y Verificación $(\mathrm{M} \& \mathrm{~V})$ es un proceso que consiste en realizar de manera correcta medidas de consumo energético, para establecer de forma fiable el ahorro real generado en una instalación dentro de un programa de gestión energética.

El ahorro de energía no se puede medir de forma directa ya que representa la ausencia del consumo de energía. Por este motivo, el ahorro se determina comparando el consumo, o la demanda, antes y después de la implementación de un proyecto de eficiencia energética, al tiempo que se realizan los ajustes según la variación de las condiciones iniciales.

El consumo energético del secadero se realizó tomando la lectura del caudalímetro de gas natural instalado en el secadero. Esta lectura se realizó en pequeños intervalos de tiempo, con el fin de registrar las incidencias producidas, y siempre en intervalos de tiempo total, superiores a 3 horas.

Además de la lectura del consumo en el caudalímetro de gas se anotó la presión y temperatura del gas natural en el interior de la conducción, mediante estos datos es posible normalizar el caudal de gas, mediante la siguiente expresión:

$$
\mathbf{Q}_{\mathbf{N}}=\mathbf{Q} \cdot\left(\frac{\mathbf{T}_{0}}{\mathbf{T}_{\text {gas }}}\right) \cdot\left(\frac{\mathbf{P}_{\text {gas }}+\mathbf{P}_{\text {atm }}}{\mathbf{P}_{0}}\right) \quad \text { Ecuación } 1
$$

Donde:

Q: $\quad$ es el caudal obtenido a partir de la lectura directa del caudalímetro $\left(\mathrm{m}^{3} / \mathrm{s}\right)$.

$\mathrm{T}_{\text {gas: }}$ : es la temperatura del gas natural en la conducción (K).

$\mathrm{T}_{0}$ : $\quad$ es la temperatura de referencia $(273 \mathrm{~K})$.

$\mathrm{P}_{\text {gas }}$ : es la presión del gas en la conducción $\left(\mathrm{N} / \mathrm{m}^{2}\right)$.

$\mathrm{P}_{\mathrm{atm}}$ : es la presión atmosférica media $\left(\mathrm{N} / \mathrm{m}^{2}\right)$.

$\mathrm{P}_{0}: \quad$ es la presión de referencia $\left(1,013 \cdot 10^{5} \mathrm{~N} / \mathrm{m}^{2}\right)$.

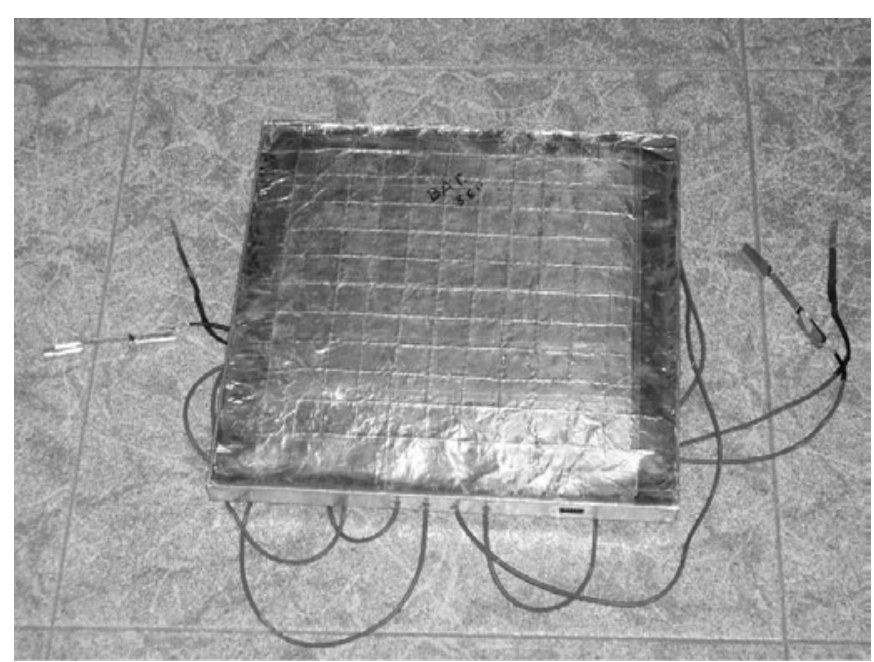

Figura 7. Dispositivo empleado para determinar el ciclo de secado.

\subsection{Determinación de las condiciones de operación del secadero}

Con el fin de validar que el sistema de recuperación de calor no afecta a las condiciones de la operación de secado se determinó experimentalmente la distribución de temperatura en interior del secadero, en las diferentes situaciones analizadas, y las propiedades de los gases de secado en la chimenea de salida del secadero.

La determinación experimental del ciclo de secado se llevó a cabo mediante un dispositivo mostrado en la figura 7 que consiste en un recipiente metálico, de dimensiones 33 $\mathrm{cm} \times 33 \mathrm{~cm}, 2,5 \mathrm{~cm}$ de espesor, recubierto externamente con fibra cerámica aislante. En su interior está situado el sistema de adquisición de datos, donde se conectan 4 termopares de tipo K. Junto a la electrónica de adquisición de datos, se sitúan dos bolsas de agua, que se congelan antes de iniciar el ensayo, para asegurar que el sistema de adquisición de datos no supera los $50{ }^{\circ} \mathrm{C}$.

La puesta en marcha y detención de la adquisición de datos, así como la descarga de los valores almacenados, se realiza a través de un ordenador, utilizando un software específico.

La determinación de los caudales gaseosos se realizó con un tubo de pitot tipo S, según la norma UNE 77225:2000.

\section{RESULTADOS OBTENIDOS}

Se realizó un estudio preliminar del funcionamiento de la instalación con el producto mayoritario. La toma de las mediciones con recuperación y sin recuperación de calor se ha realizado en condiciones de producción similares, con el objetivo de que la comparativa sea aceptada y se tenga en cuenta los criterios del IPMVP.

A continuación se exponen los resultados obtenidos en las dos situaciones de funcionamiento analizadas. 
TABLA 2. PARÁMETROS DE FUNCIONAMIENTO DEL SECADERO SIN Y CON RECUPERACIÓN.

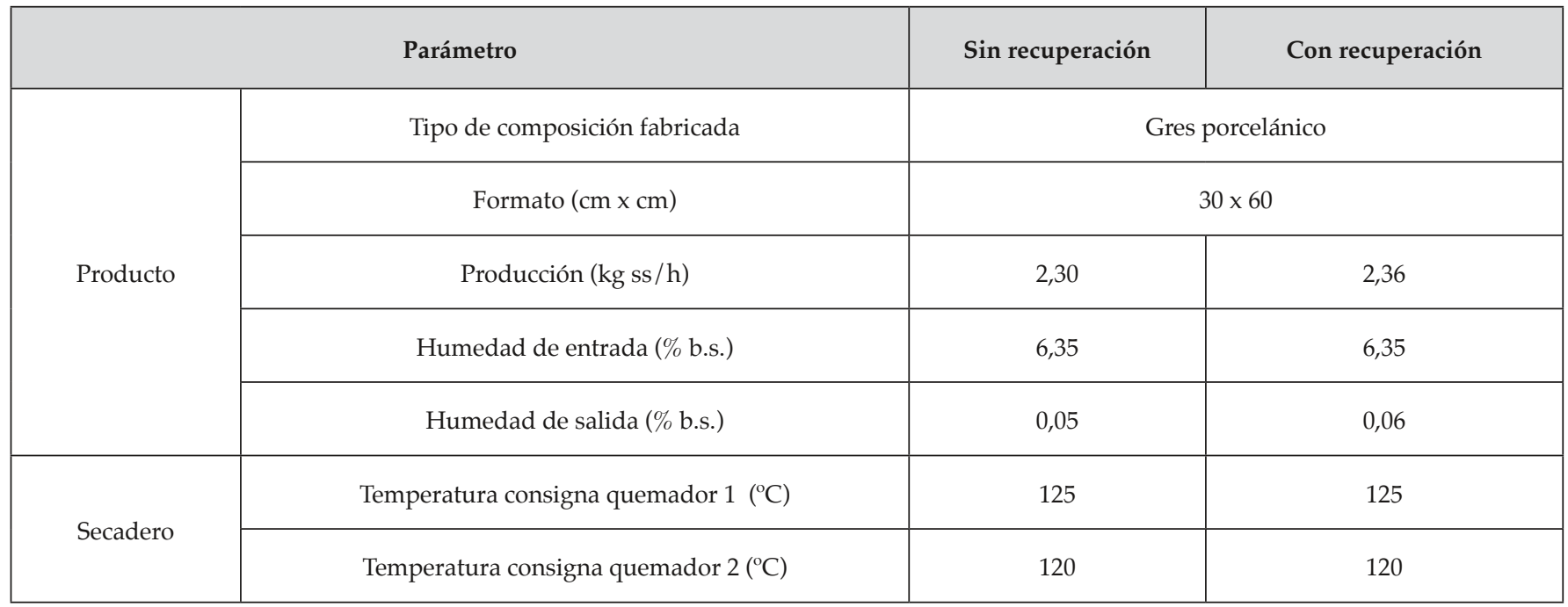

TABla 3. CARACTERIZACIÓN DE LOS GASES DE CHIMENEA DEL SECADERO SIN Y CON RECUPERACIÓN DE CALOR.

\begin{tabular}{|c|c|c|}
\hline Parámetro & Sin recuperación & 120 \\
\hline Temperatura $\left({ }^{\circ} \mathrm{C}\right)$ & 120 & \\
\hline $\begin{array}{c}\text { Humedad absoluta } \\
(\mathrm{kg} \text { agua } / \mathrm{kg} \text { aire seco })\end{array}$ & 0,100 & 0,090 \\
\hline Caudal de gases $\left(\mathrm{m}_{\mathrm{N}}^{3} / \mathrm{h}\right)$ & 7800 & 7900 \\
\hline
\end{tabular}

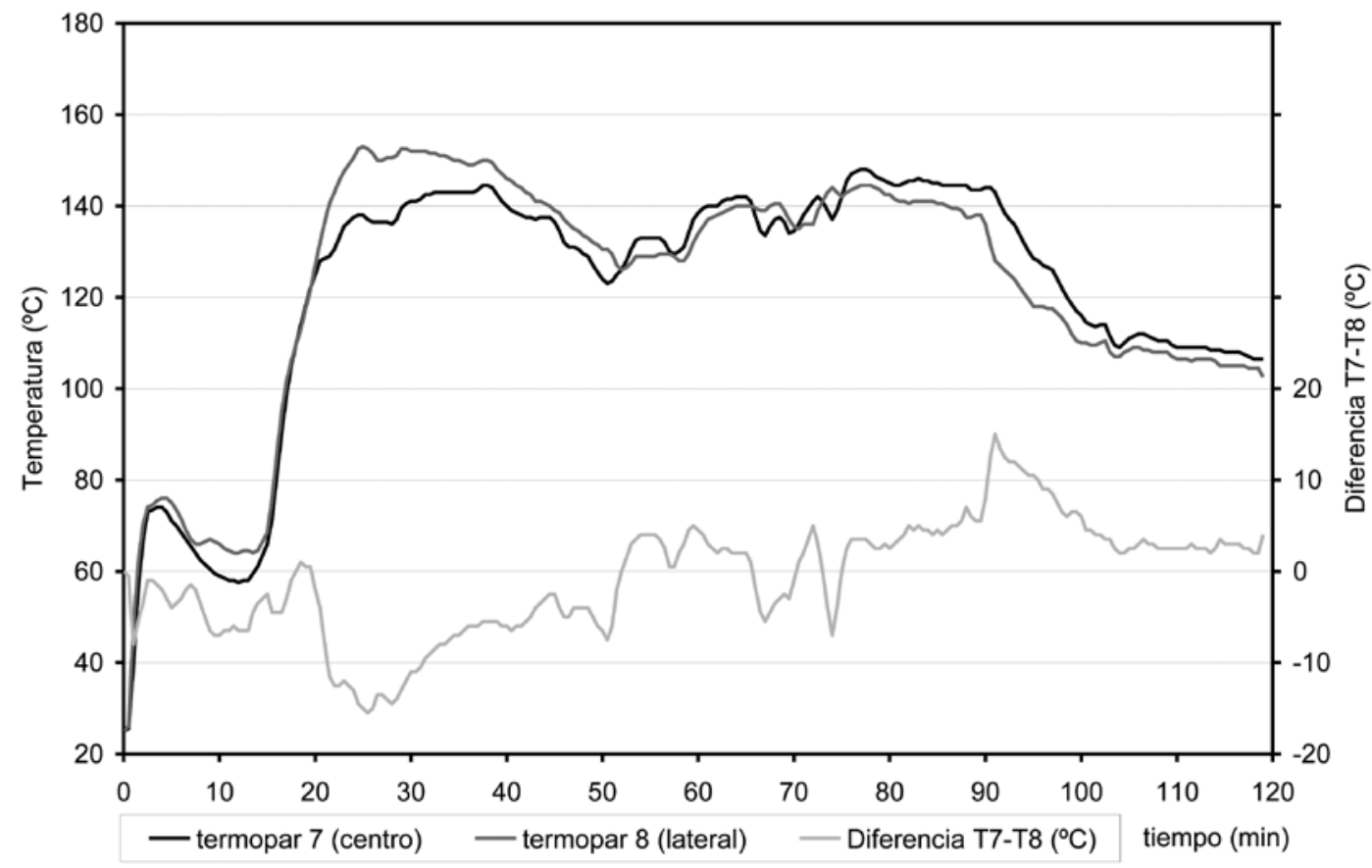

Figura 8. Temperatura de gases de secado sin recuperación. 
TABLA 4. AHORRO ENERGÉTICO DEL SECADERO CON RECUPERACIÓN.

\begin{tabular}{|c|c|c|}
\hline Parámetro & Sin recuperación & Con recuperación \\
\hline Consumo de gas natural $\left(\mathrm{m}^{3}{ }_{\mathrm{N}} / \mathrm{h}\right)$ & 74 & 22 \\
\hline Consumo PCS $(\mathrm{KW})$ & 870 & 70 \\
\hline Ahorro energético $(\%)$ & --- & 650 \\
\hline Reducción emisiones $\left(\mathrm{t} \mathrm{CO}_{2} / \mathrm{año}\right)$ & -- & 70 \\
\hline
\end{tabular}

En la tabla 2 se muestran los parámetros de funcionamiento del secadero estudiado en las situaciones analizadas.

Con la finalidad de validar el funcionamiento del sistema de recuperación de calor, se analizaron las propiedades de los gases de secado mientras se estaba recuperando calor desde los hornos y sin la recuperación, con el fin de comprobar si éstas se ven afectadas por el sistema de recuperación empleado.

En la tabla 3 se muestran las propiedades de los gases de chimenea del secadero en las situaciones analizadas.

Se observa que las condiciones de los gases de secado apenas se ven modificadas por el sistema de recuperación empleado.

La menor humedad absoluta de los gases de secado, con el sistema de recuperación de calor, se corresponde con el menor consumo de gas natural de secadero, ya que uno de los productos de la combustión del gas natural es el vapor de agua.

El ciclo de secado experimental se llevó a cabo utilizando el dispositivo indicado en el apartado 4.2. En la figura 8 y en la figura 9 se muestran los resultados obtenidos, sin recuperación y con recuperación de calor, respectivamente.

Los resultados obtenidos muestran que no existen diferencias de temperaturas importantes entre la zona central y lateral del secadero. Durante el tramo ascendente, la temperatura de los gases en el lateral del secadero es ligeramente superior que en el centro; en ambas situaciones, esta situación se invierte durante el tramo descendente. La máxima diferencia de temperatura observada asciende a 15 ${ }^{\circ} \mathrm{C}$, aproximadamente.

Se observa que cuando la recuperación de calor está en funcionamiento, se alcanzan temperaturas más elevadas en la segunda mitad del ciclo de secado.

El ciclo de secado es, formalmente, similar en ambas situaciones, por lo que puede concluirse que el modo de recuperación de calor procedente del horno apenas incide sobre el ciclo de secado.

En la tabla 4 se muestra el ahorro energético alcanzado en el secadero en las condiciones de trabajo establecidas, tras instalar el sistema de recuperación de calor.

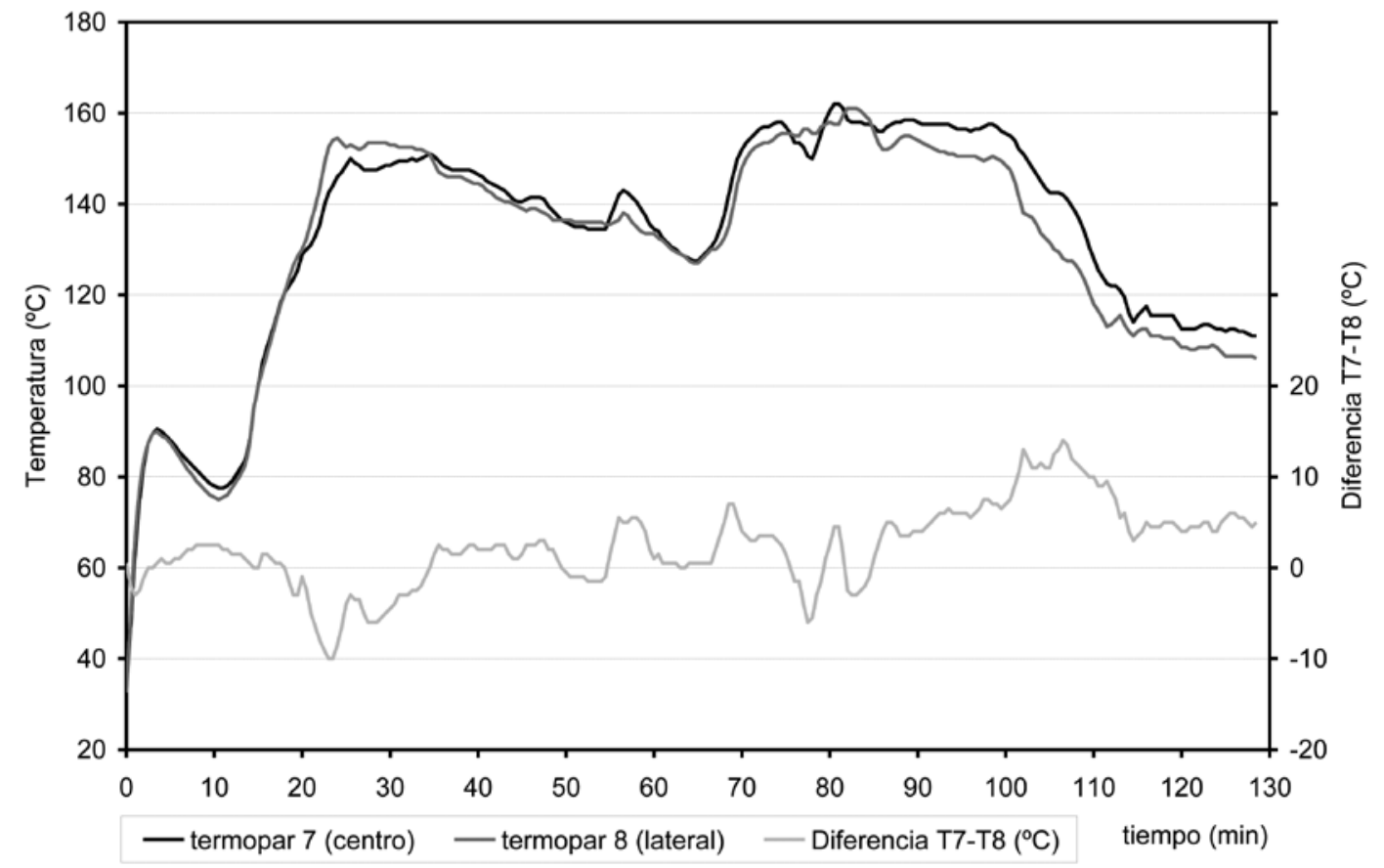

Figura 9. Temperatura de los gases con recuperación. 


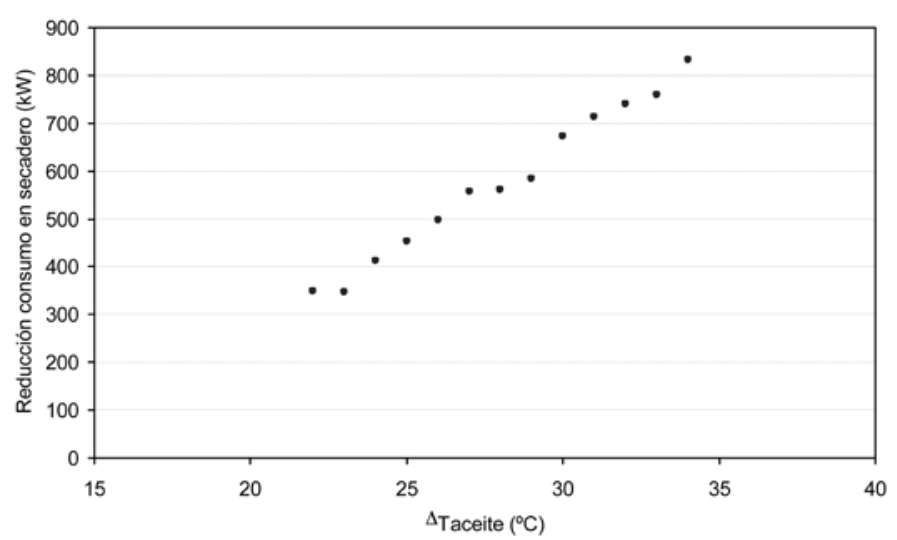

Figura 10. Ahorro energético en el secadero en función del salto térmico del aceite.

Se observa una reducción en el consumo de gas natural del $70 \%$, lo que implica una reducción de las emisiones de $650 \mathrm{tCO}_{2} /$ año.

El ahorro energético en el secadero está relacionado con el salto térmico que se consiga en el aceite térmico en los intercambiadores del horno (diferencia entre la temperatura de entrada y de salida del aceite): a mayor salto térmico mayor ahorro, tal y como se observa en la figura 10. En condiciones promedio de funcionamiento, el salto térmico que se consigue en el horno se sitúa en el intervalo entre 28 y $30^{\circ} \mathrm{C}$, con lo que se consigue una potencia promedio recuperada de las salidas del horno entre 600 y $700 \mathrm{~kW}$.

\section{CONCLUSIONES}

Las principales conclusiones del estudio realizado son las siguientes:

- Se ha puesto a punto una instalación experimental de recuperación de calor que permite la recuperación simultánea de energía desde la chimenea de los gases de combustión y de enfriamiento en una única corriente, utilizando un aceite térmico.

- Se ha comprobado que la instalación de este sistema no modifica las condiciones de operación ni del horno ni del secadero, de manera que no se modifican las propiedades del producto procesado.
- Los estudios realizados muestran que con este sistema de recuperación de calor es posible obtener una reducción media en el consumo de gas natural en el secadero entre $600 \mathrm{~kW}$ y $700 \mathrm{~kW}$, en función del material procesado y de las condiciones de trabajo del horno y el secadero.

\section{REFERENCIAS BIBLIOGRÁFICAS}

(1) Decisión de la Comisión 2007/589/CE, de 18 de Julio de 2007, por la que se establecen directrices para el seguimiento y la notificación de las emisiones de gases de efecto invernadero de conformidad con al Directiva 2003/87/ CE del Parlamento Europeo y del Consejo.

(2) Directiva 2003/87/CE del Parlamento Europeo y del Consejo, de 13 de octubre de 2003, por la que se establece un régimen para el comercio de derechos de emisión de gases de efecto invernadero en la Comunidad y por la que se modifica la Directiva 96/61/CE del Consejo.

(3) Directiva 2009/29/CE del Parlamento Europeo y del Consejo, de 23 de Abril de 2009, por la que se modifica la Directiva 2003/87/CE, para perfeccionar y ampliar el régimen comunitario de comercio de derechos de emisión de gases de efecto invernadero.

(4) A. ESCARDINO, El esfuerzo en innovación de la industria cerámica de la Comunidad Valenciana para reducir las emisiones de dióxido de carbono. EN: Simposio internacional sobre el cambio climático, desde la ciencia a la sociedad. Valencia: Generalitat Valenciana, 2005. pp 121-133.

(5) A. ESCARDINO; J.C. JARQUE; A. MORENO; J. DE LA TORRE, Secado de materiales cerámicos (I). Consideraciones generales. Isotermas de equilibrio. Técnica Cerámica, 185, 452-462, 1990

(6) A. ESCARDINO; J.C. JARQUE, A. MORENO; J. DE LA TORRE, Secado de materiales cerámicos (II). Cinética del secado de piezas cerámicas de pavimento y revestimiento. Modelos dinámicos. Técnica Cerámica, 190, 34-42,1991.

(7) A. ESCARDINO; Ma.A. GALLÉGO; M.J. IBÁÑEZ; F. NEGRE, Secado de materiales cerámicos. I. Factores que afectan a la humedad de equilibrio. Bol. Soc. Esp. Ceram. Vidr., 26(6), 367-373, 1987.

(8) G. MALLOL; A. MEZQUITA; D. LLORENS; J.C. JARQUE; J. SAHÚN; F VALLE, Estudio de la operación de secado en los soportes de las baldosas cerámicas en secaderos verticales. Cerámica Información, 287, 81-93, 2002.

(9) A. MEZQUITA; E. MONFORT; V. ZAERA, Sector azulejero y comercio de emisiones: reducción de emisiones de $\mathrm{CO}_{2}$, benchmarking europeo. Bol. Soc. Esp. Ceram. Vidr., 48(4), 211-222, 2009.

(10) E. MONFORT; A. MEZQUITA; R. GRANEL; E. VAQUER; A. ESCRIG; A. MIRALLES; V. ZAERA, Análisis de consumos energéticos y emisiones de dióxido de carbono en la fabricación de baldosas cerámicas. Bol. Soc. Esp. Ceram. Vidr., 49(4), 303-301, 2010.

(11) E. MONFORT; I. CELADES; S. GOMAR; F. RUEDA; J. MARTÍNEZ, Characterisation of acid pollutant emissions in ceramic tile manufacture. Bol. Soc. Esp. Ceram. Vidr., 50(4), 179-184, 2011.

\section{AGRADECIMIENTOS}

El estudio presentado ha sido financiado por el Instituto de la Mediana y Pequeña Industria Valenciana (IMPIVA) dentro del Programa de investigación y Desarrollo Tecnológico a través del proyecto IMIDTF/2010/179. 